SCIENTIFIC LETTER

\title{
Perioperative serum inflammatory response and the development of atrial fibrillation after coronary artery bypass surgery
}

\author{
J Cosgrave, J B Foley, R Kelly, E McGovern, K Bennett, V Young, M Tolan, P Crean, D Kelleher, \\ M J Walsh
}

$\Lambda$ trial fibrillation (AF) remains the most common cardiac arrhythmia and is associated with an increase in both morbidity and mortality. Some data in both the surgical and non-surgical settings suggest that inflammation has a pivotal role in the onset and propagation of AF. As on pump coronary artery bypass surgery is associated with a significant inflammatory response and an incidence of AF between $11-40 \%$, we decided to use this model to study if there was a potential association between perioperative response and the development of postoperative AF. ${ }^{12}$

\section{PATIENTS AND METHODS}

The study was approved by the hospital ethics committee and all patients provided informed consent. This study complies with the Declaration of Helsinki. We recruited 149 nondiabetic patients undergoing first time non-emergency on pump coronary artery bypass grafting. The exclusion criteria were impaired left ventricular function (ejection fraction $<40 \%$ ), renal failure (creatinine $>200 \mu \mathrm{mol} / \mathrm{l}$ ), malignancy, chronic inflammatory conditions, and concurrent infection.

Patients' cardiac rhythm was monitored continuously for 72 hours after surgery, which was reviewed by a cardiologist daily. The primary end point was defined as new onset AF that persisted for at least one hour or that required urgent treatment because of instability.

High sensitivity $\mathrm{C}$ reactive protein was measured by rate nephelometry with a Dade Behring apparatus. The adhesion molecules P-selectin, E-selectin, soluble intercellular adhesion molecule 1 (sICAM-1), and soluble vascular cell adhesion molecule 1 (sVCAM-1) were measured in stored sera by an enzyme linked immunosorbent assay technique. The kits were supplied by R\&D Systems (Abingdon, UK). Results were corrected for haemodilution by packed cell volume by the following formula: result $\times$ baseline packed cell volume / postoperative packed cell volume.

During the initial phase of the study (100 patients) blood was drawn just before anaesthetic induction and 6, 24, 48, and 72 hours after the onset of cardiopulmonary bypass. In the second phase (49 patients) baseline samples were obtained as above and the postoperative samples were taken 72 hours after the onset of cardiopulmonary bypass. This time was chosen based on our initial data from the first 100 patients and the fact that postoperative AF is most common on the third day after surgery. Samples were immediately centrifuged and stored at $-70^{\circ} \mathrm{C}$.

Means (SD) are presented for normal data, medians and interquartile ranges for non-normal data, and proportions for categorical data. For the patients with multiple time points the postoperative inflammatory response was assessed by calculating the area under the curve for the inflammatory markers. Patients who developed AF and those who did not were compared by a Student's $t$ test for normally distributed continuous data, by Wilcoxon rank sums or a Kruskal-Wallis test for non-normal continuous data, and by $\chi^{2}$ test for categorical data. A p $<0.05$ was considered significant, with two sided tests. Data were analysed by the JMP IN software (SAS Institute Inc, Cary, North Carolina, USA).

\section{RESULTS}

Fifty five patients (37\%) developed AF and there were no significant demographic differences between the two groups apart from the patients who developed AF being older $(65.2$ (8) years compared with 60.9 (10) years in the non-AF group, $\mathrm{p}=0.007$ ) and having a longer hospital stay.

Surgery was associated with an inflammatory response in all patients as reflected by an increase in the inflammatory markers from baseline (table 1).

In the initial phase of 100 patients, 39 developed AF. When analysing the data the area under the curve of the postoperative inflammatory markers was compared. The AF and non-AF groups did not differ in sE-selectin $(\mathrm{p}=0.19)$, sPselectin $(p=0.43), \quad$ sICAM-1 $\quad(p=0.94), \quad$ sVCAM-1 $(p=0.62)$, or $C$ reactive protein $(p=0.79)$ concentrations. Adjustment of these data by logistic regression for age and baseline values did not alter the significance of the results.

In the second phase of the study, 49 patients were recruited; 16 of these (33\%) developed AF. We combined the data from the two phases and looked at the inflammatory markers at baseline and day 3 postoperatively. The median values at baseline and on day 3 are presented (table 1). The $55(37 \%)$ patients who developed AF and the 94 who did not did not differ in the various inflammatory markers either in absolute terms or for the degree of change from baseline.

\section{DISCUSSION}

We have studied the potential association between the perioperative inflammatory response, assessed by serum inflammatory markers, and postoperative AF. Although coronary artery bypass surgery was associated with a major inflammatory response we found no difference in the degree of the inflammatory response between patients who did and those who did not develop AF.

The potential role of inflammation in the pathogenesis of AF has been proposed with varying data to support it. ${ }^{3}$ The best evidence comes from work on polymorphisms of the promoter sequence of the interleukin 6 gene. ${ }^{4}$ In this study 110 patients were recruited. Twenty six developed AF, of whom 21 had the GG phenotype, compared with 41 in the non-AF group. This gave a hazard ratio of 3.25 for the development of AF. Interestingly, the AF group had higher

Abbreviations: $A F$, atrial fibrillation; sICAM-1, soluble intercellular cell adhesion molecule 1; sVCAM-1, soluble vascular cell adhesion molecule 1 
Table 1 Baseline and day 3 inflammatory markers (corrected for baseline) in 149 patients with and without atrial fibrillation (AF)

\begin{tabular}{|c|c|c|c|}
\hline & AF $(n=55)$ & Non-AF $(n=94)$ & p Value \\
\hline \multicolumn{4}{|l|}{ Baseline } \\
\hline $\mathrm{sE}$-selectin (ng/ml) & $41.9(33.2-55.1)$ & $48.5(34.6-63.9)$ & 0.07 \\
\hline sP-selectin (ng/ml) & $106.8(69-144)$ & 105 (77.5-133.7) & 0.98 \\
\hline sICAM-1 (ng/ml) & $284(239-322)$ & 282 (233-343) & 0.73 \\
\hline sVCAM-1 (ng/ml) & $465(367-596)$ & 438 (335-574) & 0.29 \\
\hline CRP (mg/l) & $25.7(11.9-40.9)$ & $33.5(14.5-62.8)$ & 0.54 \\
\hline \multicolumn{4}{|l|}{ Day 3} \\
\hline $\mathrm{sE}$-selectin $(\mathrm{ng} / \mathrm{ml})$ & 56.3 (41.9-71.9) & $58.7(41-76.6)$ & 0.66 \\
\hline $\mathrm{sP}$-selectin $(\mathrm{ng} / \mathrm{ml})$ & $116(93.9-153.6)$ & $130.3(93.2-179)$ & 0.35 \\
\hline sICAM-1 (ng/ml) & 486 (399-597) & $510(394-616)$ & 0.80 \\
\hline sVCAM-1 (ng/ml) & 706 (538-869) & $653(517-834)$ & 0.17 \\
\hline CRP (mg/l) & 2440 (1710-2900) & $2600(1860-3070)$ & 0.34 \\
\hline \multicolumn{4}{|l|}{ Corrected values } \\
\hline $\mathrm{sE}$-selectin (ng/ml) & $10.9(0.153-24.8)$ & $7.62(-2.7-20.5)$ & 0.33 \\
\hline sP-selectin (ng/ml) & $20.3(-20.8-40.2)$ & $17.63(-5.1-61.6)$ & 0.37 \\
\hline sICAM-1 (ng/ml) & $180(119-290)$ & $201(131-297)$ & 0.95 \\
\hline sVCAM-1 (ng/ml) & $232(87-356)$ & $197(92-344)$ & 0.55 \\
\hline CRP (mg/l) & 2410 (1700-2870) & $2540(1830-3060)$ & 0.32 \\
\hline
\end{tabular}

Data presented are medians and interquartile ranges. Comparison by a Wilcoxon rank sum test.

CRP, C reactive protein; $s$, soluble; sICAM, soluble intercellular adhesion molecule 1 ; sVCAM, soluble vascular cell adhesion molecule 1 .

concentrations of interleukin 6 and fibrinogen but not of $\mathrm{C}$ reactive protein.

Although we did not see a relation between the inflammatory markers that we assessed and postoperative AF, we cannot exclude the possibility that inflammation may still have an important role in the pathogenesis of AF. Serum markers, although useful, are surrogates and do not provide exact information on the degree of atrial inflammation associated with the surgery and do not provide information about the site of the inflammation. The extent of inflammation and regions of the atria that are inflamed may have relevance in the development of postoperative AF. Details of the degree of pericardial inflammation, the extent of transmurality of the process, and the regional variability in inflammation with involvement of key arrhythmogenic areas such as the pulmonary veins cannot be assessed by the serum markers we used. Radioactively labelled inflammatory cells may provide more concise information in this regard.

\section{ACKNOWLEDGEMENTS}

We acknowledge the financial support of the Royal City of Hospita Trust Fund for our project, for the continued assistance of all the staf of the Keith Shaw cardiothoracic surgery unit, and Professor Dermot Kelleher and the staff of the Sir Patrick Duns research laboratory. We also thank Ms Carole Schilling RGN for her enthusiastic support throughout this project.

\section{Authors' affiliations}

J Cosgrave, J B Foley, R Kelly, P Crean, D Kelleher, M J Walsh, Department of Cardiology, St James's Hospital, Dublin, Ireland E McGovern, V Young, M Tolan, Department of Cardio-Thoracic surgery, St James's Hospital

K Bennett, Department of Pharmacology and Therapeutics, St James's Hospital

This work was supported by a grant from The Royal City Of Dublin Hospital trust fund.

There are no potential conflicts of interest.

Correspondence to: Dr Brendan Foley, CREST Directorate, St James's Hospital, James's Street, Dublin 8, Ireland; foleysec@stjames.ie

Accepted 10 February 2005

\section{REFERENCES}

1 Ommen SR, Odell JA, Stanton MS. Atrial arrhythmias after cardiac surgery. N Engl J Med 1997;336:1429-34

2 Edmunds LH. Inflammatory response to cardiopulmonary bypass. Ann Thorac Surg 1998;66:S12-6.

3 Karantzopoulos $\mathbf{P}$, Kolettis T, Siogas K, et al. Atrial fibrillation and electrical remodelling: the potential role of inflammation and oxidative stress. Med Sci Monit 2003;9:RA225-9.

4 Gaudino M, Andreotti F, Zamparelli R. The -174 G/C interleukin-6 polymorphism influences postoperative interleukin- 6 levels and postoperative atrial fibrillation: is atrial fibrillation an inflammatory complication? Circulation 2003;108(suppl II):I1195-9.

\section{1 th European Forum on Quality Improvement in Health Care}

26-28 April 2006, Prague, Czech Republic

For further information please go to: www.quality.bmipg.com

Book early to benefit from a discounted delegate rate 